\title{
Dopa-responsive dystonia with additional unusual clinical features: A case report confirmed by molecular genetics
}

\author{
Woong-Woo Lee ${ }^{1 \oplus}$, Jong-Moon Choi ${ }^{2 \oplus}$, and Cha Gon Lee ${ }^{3, * \oplus}$ \\ 'Department of Neurology, Nowon Eulji Medical Center, Eulji University, Seoul, Korea \\ ${ }^{2}$ Department of Laboratory Medicine, GC Genome, Yongin, Korea \\ ${ }^{3}$ Department of Pediatrics, Nowon Eulji Medical Center, Eulji University, Seoul, Korea
}

The term dopa-responsive dystonia (DRD) is used to describe a group of neurometabolic disorders, which are characterized by dystonia, and are typically associated with diurnal fluctuations and respond to levodopa treatment. Autosomal dominant DRD (DYT5a, MIM\# 128230) is caused by a heterozygous mutation in the GTP cyclohydrolase 1 (GCH1) gene (MIM\# 600225). GCH1 encodes an enzyme, which is involved in the biosynthesis of tetrahydrobiopterin, an essential co-factor for tyrosine hydroxylase. Herein, we report the case of a 16-year-old girl who was diagnosed with DYT5a. She exhibited additional unusual clinical features, including intellectual disability, depression, multiple skeletal anomalies, and short stature, which are not commonly observed in patients with DYT5a. The patient harbored a heterozygous missense variant, c.539A >C, p.GIn180Pro, in the GCH1 gene, which was identified by targeted gene panel analysis using next-generation sequencing.

Key words: Dystonia, Dopa-responsive, Autosomal dominant, GCH1, Intellectual disability, Depression.

\section{Introduction}

The term dopa-responsive dystonia (DRD) is used to describe a group of rare, inherited neurometabolic disorders of the dopamine synthesis pathway. These disorders were initially recognized and as 'hereditary progressive basal ganglia disease' by Segawa [1] in 1971. DRD is characterized by early-onset lower limb dystonia that causes gait disturbance and diurnal fluctuations, and responds to an excellent response to levodopa treatment [2]. The precursor of the neurotransmitter dopamine is synthesized from tyrosine by the enzyme tyrosine hydroxylase, which uses tetrahydrobiopterin (BH4) as a cofactor. DRD is caused by mutations in genes that encode the enzymes involved in the biosynthesis of levodopa, and the features of DRD can range from mild to severe $[2,3]$. Autosomal dominant DRD (DYT5a, MIM \# 128230) is caused by heterozygous mutations in the GCH1 gene (MIM \#600225), which is located on chromosome 14q13. It is the most common cause of DRD [2,3]. Autosomal recessive DRD (DYT56, MIM \#605407), caused by mutations in the TH gene (MIM\# 191290), and DRD due to sepiapterin reductase deficiency (SRD, MIM \#612716), caused by mutations in the SPR gene (MIM\# 182125), are less common. The GCH1 gene encodes an enzyme known as GTP cyclohydrolase I (GTPCH1), which is needed for $\mathrm{BH} 4$ synthesis. Thus, mutations in the $\mathrm{GCH} 1$ gene disrupt the production of $\mathrm{BH} 4$, leading to a decrease in dopamine levels [3].

Received: 23 December 2019, Revised: 21 January 2020, Accepted: 1 February 2020, Published: 30 June 2020

${ }^{*}$ Corresponding author: Cha Gon Lee, M.D., Ph.D. (iD https://orcid.org/0000-0001-7294-229X

Division of Child Neurology, Department of Pediatrics, Nowon Eulji Medical Center, Eulji University, 68 Hangeulbiseok-ro, Nowon-gu, Seoul 01830, Korea. Tel: +82-2-970-8222, Fax: +82-2-970-0068, E-mail: leechagon@eulji.ac.kr

Conflict of interest: The authors declare that they do not have any conflicts of interest.

(ㄷ) This is an open-access article distributed under the terms of the Creative Commons Attribution Non-Commercial License (http://creativecommons.org/licenses/by-nc/4.0/) which permits unrestricted non-commercial use, distribution, and reproduction in any medium, provided the original work is properly cited.

(c) Copyright 2020 by the Korean Society of Medical Genetics and Genomics 
Herein, we report the case of a girl who harbored a heterozygous missense variant, c.539A >C, p.GIn180Pro, in GCH1, which was identified by targeted gene panel analysis using nextgeneration sequencing (NGS). The patient exhibited additional unusual clinical features of DYT5a, including mild intellectual disability, depression, multiple skeletal anomalies, and short stature.

\section{Case}

The Institutional Review Board of Nowon Eulji Medical Center (approval number: EMCS 2018-11-035) approved the use of human clinical materials and blood in this study. Written informed consent for the publication of medical images and genetic test results was obtained from the patient and her guardian.

A 16-year-old girl visited our clinic in November 2018 with the chief complaint of gait unsteadiness since childhood. The patient experienced diurnal fluctuations in gait unsteadiness that progressively worsened over time. The family history was not relevant for neurodevelopmental disorders. The pedigree chart is presented in Fig. 1A. Her family reported that she exhibited psychomotor developmental delays since early childhood. She had mild intellectual disability with a Full Scale intelligence quotient of 61, obtained with the Korean-Wechsler Intelligence Scale for Children III, which was used at other psychiatric hospitals when she was 13 years old. She was treated for depression with antidepressant medication by a child psychologist. She underwent physical therapy for moderate scoliosis with a 34-degree curve and wore a brace. Physical examination revealed that the patient was short in stature, with a height of $146.3 \mathrm{~cm}(-2.52$ standard deviation, SD). She weighed $33.8 \mathrm{~kg}(-3.41 \mathrm{SD})$, and had a head circumference of $50 \mathrm{~cm}(-0.633$ SD). She presented with generalized dystonia with diurnal fluctuation, mainly in the limbs and back, which had started in the lower limbs and spread upwards with age. She had poor handwriting skills. She showed no evidence of dystonia of the eye, tongue, larynx, and neck. Neurologic examination revealed ankle clonus and brisk tendon reflexes in the lower limbs. She did not have any tremor
A
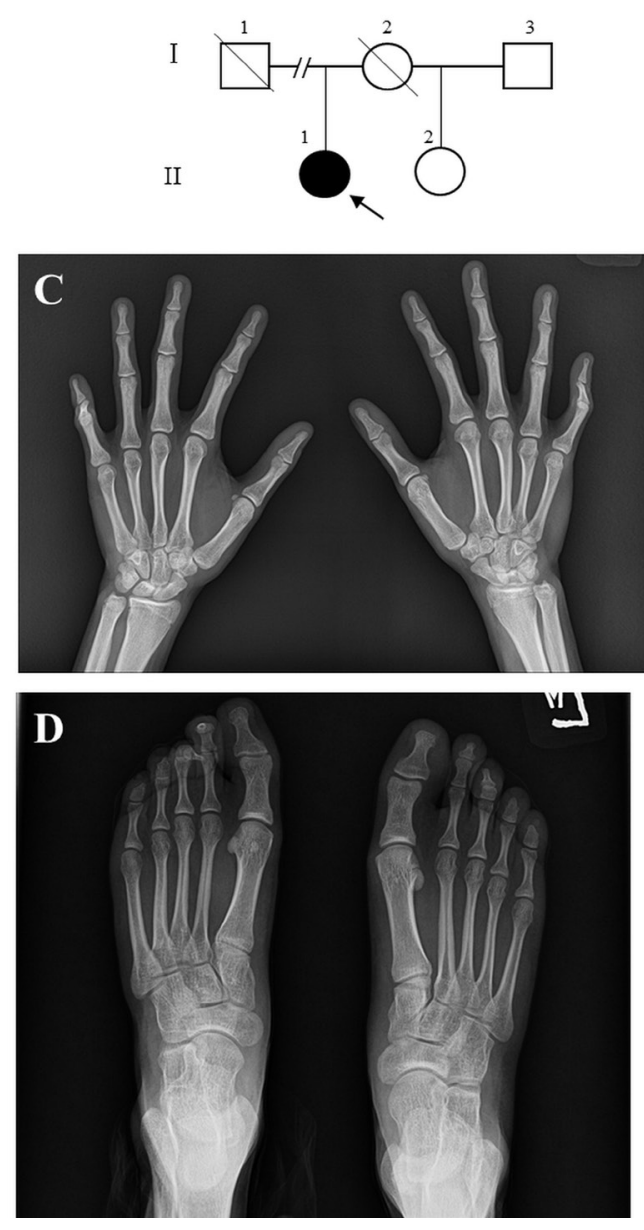

\section{B}

$R_{t}$

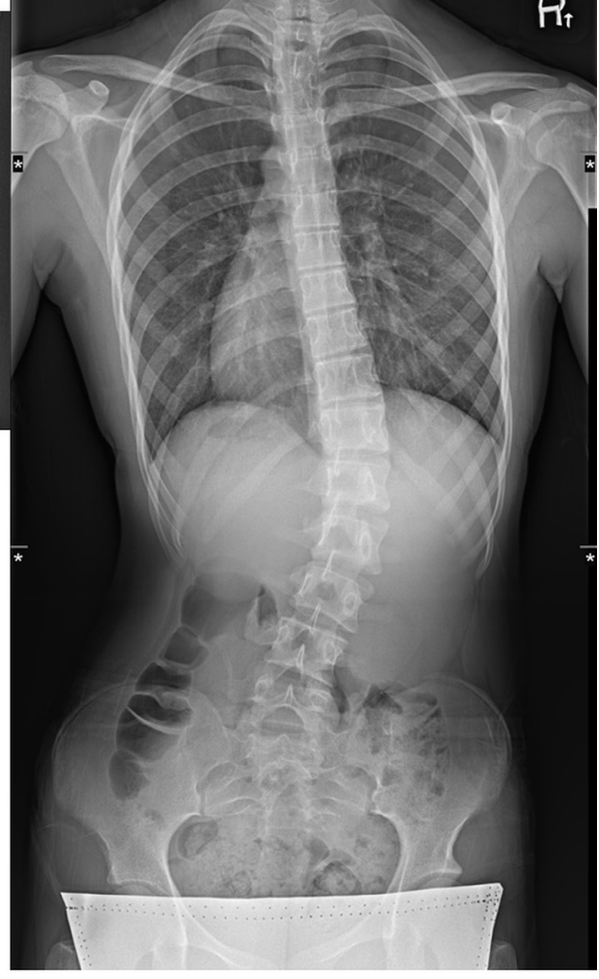

Fig. 1. (A) Family pedigree of our patient with dopamine response dystonia. (B) Spine $X$-ray shows moderate thoracolumbar scoliosis. (C) X-rays of both hands reveal mild shortening of the middle phalanges, giving a radial curvature to the 5 th fingers. (D) Flexion deformities of the distal interphalangeal joint on the 3rd distal pharynx and mild hallux valgus are observed. 
or rigidity. She had moderate thoracolumbar scoliosis, severe clinodactyly of the fifth finger, mallet toe, flat feet, and mild hallux valgus. Radiographic evaluations of the skeletal findings are presented in Fig. 1B-D. The results of laboratory tests, including pituitary function, were within the normal range. The findings of brain magnetic resonance imaging were also normal. Administration of levodopa and decarboxylase inhibitors led to the complete regression of dystonia within days, and the patient sustained a good response with a dose of $4 \mathrm{mg} / \mathrm{kg} /$ day without any side effects. Moreover, the patient's symptoms of depression improved.

Genomic DNA was extracted from the patient's peripheral blood leukocytes and underwent targeted gene panel sequencing with an xGen Inherited Diseases Panel (Integrated DNA Technologies, Inc., Coralville, IA, USA), which is designed for targeted enrichment of approximately 4,503 clinically relevant genes. Massive parallel sequencing was performed with the NextSeq platform (Illumina Inc., San Diego, CA, USA) at Green Cross Genome (Yongin, Korea). A heterozygous missense variant of $\mathrm{GCH} 1$ c.539A > C, p.GIn180Pro, was identified on the basis of the reference sequence NM_000161.2. It was classified as a likely pathogenic variant with PM 1 (mutational hot spot without benign variation), PM2 (absence from populations such as gnomeAD, ExAC, 1000 g, and KRGDB), PP3 (multiple lines of computational evidence, including SIFT, PolyPhen-2, MutationTaster, support a deleterious effect), PP4 (highly specific phenotype), and PP5 (reputable source reported as pathogenic, including PMID 19491146) according to the Standards and Guidelines for the Interpretation of Sequence Variants by the American College of Medical Genetics and Genomics and the Association for Molecular Pathology [4-6]. This variant was confirmed by Sanger sequencing, the results of which are presented in Fig. 2. There were no pathological mutations in the genes known to be associated with DRD, including the $T H$ and SPR genes. No other variants of GCH1 suggest compound heterozygosity. Copy number variant analysis using exome sequencing read depth data did not reveal any clinically significant copy number variations.

\section{Discussion}

The association of DRD with non-motor psychiatric symptoms such as anxiety, obsessive-compulsive disorder, sleep disturbances, and depression has been reported in a few patients with DYT5a $[2,7]$. Genetic mutations that alter the production of neurotransmitters and receptors can potentially affect the function of basal ganglia circuits and lead to non-motor symptoms [8]. $\mathrm{BH} 4$ is a cofactor for the conversion of phenylalanine to tyrosine and the subsequent conversion of tyrosine to levodopa. Levodopa serves as a substrate for the production of neurotransmitters such as dopamine, norepinephrine, and epinephrine. $\mathrm{BH} 4$ is also a cofactor for the conversion of tryptophan to serotonin [9]. These neurotransmitters have important functions in cognitive and affective circuits. Depression was not reported frequently among the other non-motor psychiatric symptoms of DRD $[5,10]$. Only a few studies have reported depression as a psychiatric manifestation in patients with DRD $[11,12]$. Van Hove et al. [11] reported that some patients even presented with psychiatric problems first, before the development of motor symptoms. However, it could not be concluded that depression was one of the primary symptoms of DRD. Previous reports are scarce owing to the low prevalence of this condition. Depression could be secondary to progression of motor symptoms in patients with DRD. Furthermore, earlier studies reported that most patients respond well to levodopa substitution with medication that affects serotonergic neurotransmission $[11,12]$. Our patient had been treated for depression for years. Her depressive symptoms improved significantly, upon initiation of treatment with levodopa. This improvement could also be secondary to the improvement in gait, following levodopa treatment. Nevertheless, the increased risk of developing depression due to genetic factors independent of GTP cyclohydrolase deficiency and identification of the direct effect of levodopa in patients with DRD cannot completely be ruled out.

Cognition, as opposed to affective disorder symptoms, is usually preserved in patients with DYT5a, even during advanced stages of the disease [3]. Rarer subtypes of DYT5b and SRD typically present with greater severity, including global developmental delays and cognitive impairment [3]. To date, there 
have been several reports of patients with DYT5a, who exhibited developmental delays and intellectual disabilities, but these patients had compound heterozygotes of $G C H 1[13,14]$. It has been suggested that $\mathrm{GCH} 1$ activity is involved in cognitive function and behavior in humans $[8,15]$. Interestingly, our patient exhibited mild intellectual disability. Thus, this study suggests the possibility of a wide spectrum of influence of $G C H 1$ activity on cognitive function in patients with DYT5a.

Our patient presented with short stature without other known genetic causes, based on the results of targeted gene panel analysis. Several studies have reported that patients with early childhood-onset DYT5a exhibit growth retardation [3]. However, it is uncertain whether growth retardation in patients with early childhood-onset DYT5a is the result of a genetic defect in $\mathrm{GCH} 1$ or the secondary effect of dystonia on skeletal development. Our patient had several skeletal deformities, such as scoliosis, clinodactyly, mallet toe, and hallux valgus. Scoliosis secondary to dystonia is commonly reported in patients with DRD [16]. Furthermore, an earlier case report stated that pediatric patients with scoliosis and DRD achieved rapid curve resolution after levodopa treatment [9]. Although our patient's scoliosis did not improve after levodopa treatment, we believe that it was probably a result of DYT5a. However, the possibility remains of the existence of a link between dystonia and the other minor skeletal deformities that our patient exhibited.

While it is unclear whether early diagnosis and initiation of levodopa treatment improves the prognosis and prevents the progression of unusual clinical manifestations, including intellectual disability and short stature, there is no doubt that early, accurate diagnosis is important in patients with DRD. Diagnosis of DRD can be made on clinical grounds. However, there is considerable phenotypic variability, especially in infancy and early childhood, and DRD is often misdiagnosed as cerebral palsy. Therefore, it is important that all pediatric patients with suspicion of dystonia should receive trial treatment with levodopa.

Sanger sequencing for single genes has been the gold standard test when there is a clear phenotype for a Mendelian disorder. Moreover, Sanger sequencing is more cost-effective than targeted gene panel sequencing using NGS for small-sized genes or hot spots genes. In our study, we performed target multi-gene panel sequencing as the first genetic test, because our patient showed unusual phenotypes for DRD such as intellectual disability, short stature, and multiple skeletal anomalies. Thus, we assumed that DRD was concomitant with another condition in the differential diagnosis. Single gene sequencing for $G C H 1$ is definitely the first genetic test in patients whose symptoms typically improve dramatically with low-dose administration of levodopa. We believe that targeted gene panel analysis is a useful diagnostic tool in pediatric patients with additional unusual clinical manifestations or those with an atypical phenotype that shows an incomplete response to levodopa.

In summary, we reported the case of a 16-year-old girl who was diagnosed with DRD that was confirmed through genetic sequencing. She presented with unusual clinical features including mild intellectual disability, depression, multiple skeletal anomalies, and short stature.

\section{References}

1. Segawa M. Childhood basal ganglia disease with remarkable response to L-dopa, hereditary basal ganglia disease with marked diurnal fluctuation. Shinryo (Tokyo) 1971;24:667-72.

2. Phukan J, Albanese A, Gasser T, Warner T. Primary dystonia and dystonia-plus syndromes: clinical characteristics, diagnosis, and pathogenesis. Lancet Neurol 2011;10:1074-85.

3. Segawa M. Autosomal dominant GTP cyclohydrolase I (AD GCH 1) deficiency (Segawa disease, dystonia 5; DYT 5). Chang Gung Med J 2009;32:1-11.

4. Clot F, Grabli D, Cazeneuve $C$, Roze E, Castelnau P, Chabrol B, et al. Exhaustive analysis of $\mathrm{BH} 4$ and dopamine biosynthesis genes in patients with Dopa-responsive dystonia. Brain 2009;132(Pt 7):1753-63.

5. Tassin J, Dürr $A$, Bonnet AM, Gil R, Vidailhet $M$, Lücking $C B$, et al. Levodopa-responsive dystonia. GTP cyclohydrolase I or parkin mutations? Brain 2000;123(Pt 6):1112-21.

6. Richards S, Aziz N, Bale S, Bick D, Das S, Gastier-Foster J, et al. Standards and guidelines for the interpretation of sequence variants: a joint consensus recommendation of the American College of Medical Genetics and Genomics and the Association for Molecular Pathology. Genet Med 2015;17:405-24.

7. Lee WW, Jeon BS. Clinical spectrum of dopa-responsive dystonia and related disorders. Curr Neurol Neurosci Rep 2014;14:461.

8. Sunga MA, Rosales RL. Mental dysfunctions in dystonia-plus syndromes. J Parkinsons Dis 2014:4:161-7.

9. Tsirikos Al, Carr $\sqcup$, Noordeen HH. Variability of clinical expression and evolution of spinal deformity in a family with late detection of doparesponsive dystonia. Dev Med Child Neurol 2004;46:128-37.

10. López-Laso E, Sánchez-Raya A, Moriana JA, Martínez-Gual E, Camino-León R, Mateos-González ME, et al. Neuropsychiatric symptoms and intelligence quotient in autosomal dominant Segawa disease. J Neurol 2011;258:2155-62.

11. Van Hove JL, Steyaert J, Matthijs G, Legius E, Theys P, Wevers R, et al. Expanded motor and psychiatric phenotype in autosomal dominant 
Segawa syndrome due to GTP cyclohydrolase deficiency. J Neurol Neurosurg Psychiatry 2006;77:18-23.

12. Hahn H, Trant MR, Brownstein MJ, Harper RA, Milstien S, Butler IJ. Neurologic and psychiatric manifestations in a family with a mutation in exon 2 of the guanosine triphosphate-cyclohydrolase gene. Arch Neurol 2001;58:749-55.

13. Furukawa Y, Kish SJ, Bebin EM, Jacobson RD, Fryburg JS, Wilson WG, et al. Dystonia with motor delay in compound heterozygotes for GTP-cyclohydrolase I gene mutations. Ann Neurol 1998;44:10-6.

14. Kim $R$, Jeon $B$, Lee WW. A systematic review of treatment outcome in patients with dopa-responsive dystonia (DRD) and DRD-plus. Mov Disord Clin Pract 2016;3:435-42.

15. Sadahiro $R$, Suzuki $A$, Matsumoto $Y$, Shibuya $N$, Enokido $M$, Kamata $M$, et al. Functional polymorphism of the GTP cyclohydrolase 1 gene affects the personality trait of novelty seeking in healthy subjects. Neurosci Lett 2011;503:220-3.

16. Bhandutia AK, Nangunoori $R$, Whiting DM, Sangimino MJ. Scoliosis secondary to dystonia: a case report and review of the literature. JBJS Case Connect 2017;7:e47. 